\title{
Bertrand Labasse (2020). La valeur des informations. Ressorts et contraintes du marché des idées. Presses de I'Université d'Ottawa, 420 pages.
}

Recension par Virginie Soulier, Université de Perpignan Via Domitia

L'ouvrage de Bertrand Labasse est un essai scientifique qui rend compte de la somme de ses recherches et enseignements depuis plus de 20 ans tant au Canada qu'en France. Le projet est ambitieux : proposer une théorie de la communication sur la valeur des informations, issues de tous types de médias et de textes. La question de départ : pourquoi accorder du temps et de l'attention à tel discours?

Toute production discursive cherche à "valoir »; quelles sont donc les valeurs à l'œuvre? A partir de la métaphore du "marché », l'auteur prend en compte toutes sortes d'idées et de discours pour en dégager ce qui génère de la valeur, c'est-à-dire la préférence accordée par rapport à d'autres contenus. Les principales valeurs considérées sont : d'échange, concurrentielle, attentionnelle, hédonique et symbolique. La notion de marché signifie "l'ensemble des informations qui s'offrent à être vues, entendues ou perçues d'une façon ou d'une autre, et qui sont à ce titre susceptibles d'être retenues ou rejetées » (2020, p. 18). II ne s'agit pas d'étudier des transactions marchandes, mais d'analyser les relations entre l'offre et la demande discursives et leurs variables : préférences ressenties, préférences exprimées, déterminants de la convenance. Contrairement aux concepts plus généralement convoqués en Sciences de l'information et de la communication (SIC), comme espace public ou encore discours social, cette notion permet de mettre au jour les ajustements sociocognitifs entre les espaces de production et de réception des discours, tant pour les médias traditionnels comme la télévision, que numériques. Concrètement, la tâche est d'expliquer, par exemple, pourquoi la naissance du girafon d'Avril est plus médiatisée et suivie que les craintes du FMI quant aux projets américains sur la dérèglementation bancaire, diffusées à peu près au même moment, sans forcément invoquer la désintégration du débat public ou l'hégémonie de la trivialité des médias de masse.

L'auteur examine et tisse avec minutie, selon une approche interdisciplinaire tout en s'inscrivant dans les SIC (philosophie, psychologie, 
sociologie, neurobiologie, littérature, etc.), des micro-études provenant tant de la presse, de la littérature que des musées, à partir d'une revue des écrits qui remonte aux auteurs de l'Antiquité jusqu'aux travaux les plus récents. Suivant une démarche inductive, Labasse nous conduit pas à pas, au fil des pages, avec érudition, humour, prudence et discussion scientifique, à découvrir toutes les strates d'un modèle théorique permettant de comprendre l'intérêt et l'attention portés aux discours et leur acceptabilité sociale. II identifie les déterminants des préférences accordées aux discours entre l'effort qu'ils exigent et le plaisir qu'ils procurent (pertinence cognitive), entre les prescriptions et proscriptions sociales (convenances sociales) qui régulent leur légitimité et recevabilité normative. En bref, les niveaux d'intérêts dépendent du niveau d'effort cognitif requis par rapport à la satisfaction attendue, mais aussi de facteurs sociaux. Dans une seconde partie de l'ouvrage, il met à l'épreuve ce modèle de l'adéquation sociocognitive.

L'originalité de la démarche est double: l'objet d'étude et les règles d'écriture. De nombreux travaux en SIC portent sur l'influence des médias et les impacts des discours. A partir de ce constat, Labasse propose de comprendre pourquoi les contenus qui nous plaisent, nous plaisent-ils ? Pour cela, il développe progressivement l'objet "marché d'idées ». Au-delà d'interroger les attentes et habitudes de lecture, l'enjeu est donc d'identifier les facteurs puis les ressorts des valeurs discursives sur un marché aussi vaste, en tant que phénomène communicationnel. Avec l'inflation de l'information, la fragmentation de l'offre, l'essor constant de la productivité, même la gratuité ne suffit plus à susciter de la demande. Le but est d'aider les producteurs de discours (journalistes, enseignants, écrivains) à mieux appréhender ce qui se joue sur le marché d'idées quand l'offre ne cesse de croître et les prix de baisser, alors que le temps des publics n'est pas extensible. Systématiquement, à la suite de chaque chapitre, Labasse apporte des "Précisions » sur sa méthode, en explicitant ses hésitations, en justifiant ses choix théoriques et en approfondissant les discussions. Ce format d'écriture offre un souffle de renouveau aux publications scientifiques. II permet de tracer le cheminement intellectuel du chercheur. L'ouvrage nous donne les critiques à lui renvoyer quant à l'efficacité d'écriture, mais indirectement aussi les conditions pour l'apprécier. Dit autrement, il faut prendre le temps de le lire (sans téléphone à la main pour éviter la "concurrence attentionnelle ») et s'approprier le raisonnement proposé voire raconté.

L'apport de cette théorisation est triple: 1. s'asseoir sur une revue de littérature solide où les références sont croisées, ordonnées et débattues en revenant sur les fondamentaux des SIC (comme le processus de traitement de l'information), 2. caractériser l'évolution du marché discursif contemporain et 3. produire un modèle théorique transférable à des situations variées pour saisir avec les nuances nécessaires des phénomènes communicationnels complexes. 
La générosité des corpus théoriques et empiriques peut laisser perplexe par rapport à la méthode déployée avec les «vieilles ficelles de la démarche analytique » (2020, p.3) associant des exemples disparates, sélectionnés selon leur portée heuristique, examinés dans la large lunette des sciences humaines et sociales, même si l'effort d'argumentation pour faire preuve de la validité scientifique est constamment étendu. D’un point de vue épistémologique, Labasse propose d'intégrer davantage aux SIC les approches sociales pour mieux cerner et prédire les phénomènes communicationnels. Son pari de l'interdisciplinarité est tenu, grâce à la diversité, à la complétude et à la critique des théories mobilisées. 